\title{
Doença ulceropéptica no idoso
}

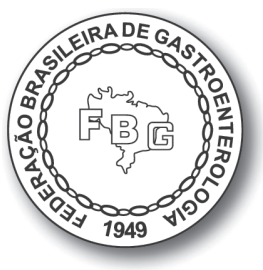

Carlos Kupski

Leonardo de Lima Lardi

\section{INTRODUÇÃO}

A doença ulcerosa péptica e suas complicações são importante causa de morbidade e mortalidade em pacientes idosos. Sabidamente, a prevalência de infecção por Helicobacter pylori $(H p)$ e o uso de anti-inflamatórios não hormonais (AINH) é elevada neste grupo de pacientes, e estas são as principais etiologias responsáveis pelo desenvolvimento desta doença. $\mathrm{O}$ diagnóstico e o tratamento adequados são de fundamental importância para controlar os sintomas, prevenir a recidiva e evitar graves complicações.

\section{ETIOLOGIA E COMPLICAÇÕES}

A infecção por $H p$ é a principal etiologia de úlceras gástricas e duodenais em todas as idades. A prevalência dessa infecção aumenta em pacientes idosos. Cerca de 60 a 70\% dos pacientes com mais de 65 anos que desenvolvem úlcera péptica são portadores de $H p$, e sua erradicação é parte fundamental da terapia para cicatrização da lesão ulcerosa.

A idade avançada é um fator de risco independente para o surgimento de complicações gástricas relacionadas ao uso de AINH. Este risco eleva-se ainda mais em pacientes com história prévia de doença péptica, com doenças cardiovasculares e naqueles que fazem uso concomitante de corticosteroides ou de anticoagulantes. Ainda assim, frequentemente pacientes com estes fatores de risco fazem uso dessas medicações, tornando elevado o risco do desenvolvimento de doença ulcerosa péptica. 
Em pacientes idosos, outro fator importante na fisiopatologia da doença péptica é a redução na secreção de fatores protetores, como muco e bicarbonato, tornando a mucosa gástrica mais suscetível a lesões causadas pelo ácido clorídrico. Por este motivo, pacientes com idade avançada apresentam maior risco de desenvolverem úlceras pépticas mesmo quando não infectados pelo Hp e quando não fazem uso de AINH.

Além de estarem sob maior risco para desenvolvimento de úlcera péptica, pacientes idosos apresentam maior chance de surgimento de complicações (especialmente sangramento) relacionadas à esta doença. A hemorragia pode se apresentar por meio de hematêmese, melena, enterorragia ou mesmo anemia ferropriva, sem ter sido identificada a exteriorização de sangue pelo trato gastrintestinal. Outra possível complicação da doença péptica é a perfuração, que se manifesta classicamente como quadro de abdome agudo e necessita de intervenção cirúrgica para seu tratamento.

Além da maior probabilidade do surgimento de complicações, quando estas ocorrem, costumam apresentar maior gravidade em pacientes idosos, especialmente naqueles com múltiplas comorbidades. Este dado foi comprovado em grande estudo populacional, que identificou que pacientes com mais de 80 anos apresentam 4 vezes mais chance de óbito em 30 dias após hemorragia por úlcera péptica quando comparados a pacientes com menos de 65 anos.

\section{APRESENTAÇÃO CLÍNICA E DIAGNÓSTICO}

A apresentação típica da doença péptica ulcerosa é dor epigástrica. Em pacientes idosos, entretanto, a doença manifesta-se de forma atípica: em cerca de $2 / 3$ dos casos, a queixa principal é apenas de desconforto epigástrico vago. Além disso, nesse grupo de pacientes, com maior frequência ocorrem complicações da doença (hemorragia ou perfuração) sem história prévia de dor abdominal. Deve haver, portanto, elevada suspeição da possibilidade do diagnóstico para que não haja atraso no diagnóstico, com consequente surgimento de complicações e sérios prejuízos ao paciente.

O método diagnóstico considerado padrão-ouro na avaliação da doença ulcerosa péptica é a endoscopia digestiva alta. Este exame permite avaliar a presença de úlcera e tratar suas possíveis complicações, além de possibilitar a realização de biópsias para que seja feito o diagnóstico diferencial entre doença péptica e neoplásica e para que seja avaliada a presença de infecção pelo $H p$. Não há indícios de que haja risco aumentado na realização de endoscopia digestiva em pacientes idosos, mas deve haver adequada avaliação antes, durante 
e após o procedimento para reduzir a chance de surgimento de complicações, especialmente em pacientes com múltiplas comorbidades.

\section{TRATAMENTO}

A base do tratamento da doença ulcerosa péptica consiste na prescrição de fármacos antissecretores e na retirada do agente agressor da mucosa gástrica, com a erradicação do $H p$ se presente e suspensão do uso de AINH quando possível.

A infecção pelo $H p$ pode ser identificada por biópsia realizada na endoscopia digestiva ou pelo teste da ureia expirada. Todos pacientes com úlcera péptica e infectados por esta bactéria devem receber tratamento para sua erradicação. Recentemente, o $4^{\circ}$ Consenso Brasileiro sobre a infecção pelo $H p$ recomendou que o tratamento para erradicação da bactéria seja realizado com esquema tríplice (inibidor de bomba de prótons, claritromicina e amoxicilina) por 14 dias (Tabela 1). Não foram feitas ressalvas que indicassem uso de outras medicações ou diferentes posologias em pacientes idosos. O Consenso orienta que não é necessário prosseguir com o uso de inibidor de bomba de prótons em casos de úlcera duodenal não complicada. Quando a úlcera for gástrica ou tiverem ocorrido complicações, é recomendado estender o uso desta medicação por 4 a 8 semanas.

O tratamento da úlcera secundária ao uso de AINH consiste na administração de inibidor de bomba de prótons por 4 a 8 semanas e, sempre que possível, na suspensão o uso dos anti-inflamatórios. Não há indício de que o uso de drogas antissecretoras além desse período possa acrescentar algum benefício clínico em pacientes que não farão mais uso de AINH. A indicação do uso de AINH deve sempre levar em consideração seu risco e benefício, especialmente em pacientes idosos e com história prévia de doença ulcerosa péptica. Caso a medicação não possa ser substituída por outra que não cause dano à mucosa gástrica, seu uso deve ser indicado na menor dose e pelo menor tempo possível. As drogas com inibição seletiva da COX-2 acarretam menor risco de complicações gastrintestinais em idosos, porém foram associadas ao aumento de doenças cardiovasculares e renais e, portanto, devem ser usadas de forma bastante criteriosa e após avaliação completa das comorbidades e dos riscos de cada paciente. Em pacientes que necessitem seguir uso de AINH e especialmente aqueles que tiveram úlcera complicada com hemorragia ou que não erradicaram o $H p$, o uso concomitante e por longo período de inibidor de bomba de prótons parece ser justificado para diminuir o risco de desenvolvimento de doença ulcerosa péptica em pacientes idosos. 
Tabela 1 Recomendação de tratamento de primeira linha para $\mathrm{Hp}-4^{\circ}$ Consenso Brasileiro sobre a infecção por Helicobacter pylori, 2018

\begin{tabular}{lll} 
Medicação & Dose & Tempo \\
\hline Inibidor de bomba de prótons & Plena a cada $12 \mathrm{~h}^{*}, \mathrm{VO}$ & 14 dias \\
\hline Claritromicina & $500 \mathrm{mg}$ a cada $12 \mathrm{~h}, \mathrm{VO}$ & 14 dias \\
\hline Amoxicilina & $1 \mathrm{~g}$ a cada $12 \mathrm{~h}, \mathrm{VO}$ & 14 dias \\
\hline
\end{tabular}

* Dose plena: omeprazol 20 mg; lansoprazol 30 mg; pantoprazol 40 mg; rabeprazol 20 mg; dexilansoprazol $60 \mathrm{mg}$; esomeprazol $40 \mathrm{mg}$.

\section{CONSIDERAÇÕES FINAIS}

A doença ulcerosa péptica segue com prevalência e potencial morbimortalidade elevados em idosos. Como a apresentação clínica não costuma ser típi$\mathrm{ca}$, os médicos que atendem esse grupo de pacientes devem ter elevada suspeição para diagnosticar e tratar precocemente a doença. Buscar erradicação do Helicobacter pylori e fazer uso racional de anti-inflamatórios são condutas que devem ser sempre aplicadas nesta situação.

\section{REFERÊNCIAS BIBLIOGRÁFICAS}

1. Coelho LGV, Marinho JR, Genta R, Ribeiro LT, Passos MCF, Zaterka S, et al. IV Consenso Brasileiro sobre a infecção por Helicobacter pylori. Arq. Gastroenterol. 2018;55(2):97-121.

2. Linder JD, Wilcox C. Acid peptic disease in the elderly. Gastroenterology Clinics of Norh America. 2001;30(2):363-76.

3. Nagaratnam N, Nagaratnam K, Cheuk G. Peptic ulcer disease in the elderly. In: Geriatrics disease - evaluation and management. Philadelphia: Springer; 2018.

4. Pilotto A. Aging and upper gastrointestinal disorders. Best Pract Res Clin Gastroenterol. 2004;18 Suppl:73-81.

5. Pilotto A, Franceschi M, Maggi S, Addante F, Sancarlo D. Optimal management of peptic ulcer disease in the elderly. Drugs Aging. 2010;27(7):545-58.

6. Tang RS, Wu JC. Managing peptic ulcer and gastroesophageal reflux disease in elderly Chinese patients - focus on esomeprazole. Clin Interv Aging. 2013;8:1433-43. 